\title{
New-generation vaccines against cholera
}

\author{
John Clemens, Sunheang Shin, Dipika Sur, G. Balakrish Nair and Jan Holmgren
}

\begin{abstract}
Cholera is a major global health problem, causing approximately 100,000 deaths annually, about half of which occur in sub-Saharan Africa. Although early-generation parenteral cholera vaccines were abandoned as public health tools owing to their limited efficacy, newer-generation oral cholera vaccines have attractive safety and protection profiles. Both killed and live oral vaccines have been licensed, although only killed oral vaccines are currently manufactured and available. These killed oral vaccines not only provide direct protection to vaccinated individuals, but also confer herd immunity. The combination of direct vaccine protection and vaccine herd immunity effects makes these vaccines highly cost-effective and, therefore, attractive for use in developing countries. Administration of these oral vaccines does not require qualified medical personnel, which makes their use practical—even in developing countries. Although new-generation oral cholera vaccines should not be considered in isolation from other preventive approaches, especially improved water quality and sanitation, they represent important tools in the public health armamentarium to control both endemic and epidemic cholera.
\end{abstract}

Clemens, J. et al. Nat. Rev. Gastroenterol. Hepatol. 8, 701-710 (2011); published online 8 November 2011; doi:10.1038/nrgastro.2011.174

\section{Introduction}

Cholera, an acute watery diarrheal disease caused by Vibrio cholerae O1, and less commonly V. cholerae O139, is a major global public health problem. ${ }^{1}$ Cholera causes widely publicized epidemics, often in the wake of natural disasters and other humanitarian emergencies, such as that recently reported in Haiti. ${ }^{2,3}$ The disease also occurs in an endemic form, which is less well publicized but still a major public health problem, with an especially large burden in sub-Saharan Africa and South Asia.,

More than $200 \mathrm{~V}$. cholerae serogroups, based on the somatic $\mathrm{O}$ antigen of this bacterium, have been identified but only the $\mathrm{O} 1$ and $\mathrm{O} 139$ serogroups are capable of causing epidemics of cholera. Most strains of these two serogroups produce cholera toxin, which causes the majority of symptoms seen in the disease. Other serogroups rarely produce this key virulence factor. Cholera toxin is encoded by genes derived from a filamentous, lysogenic bacteriophage, termed CTX $\varphi$ (Figure 1), which undergoes site-specific integration into the $V$. cholerae genome as a prophage. The genetic sequence including the prophage can be transmitted horizontally between bacteria, leading to formation of stable lysogens in recipient $V$. cholerae cells, and converting them from a nontoxigenic to a toxigenic form. ${ }^{6}$

$V$. cholerae $\mathrm{O} 1$ is further classified into classical and El Tor biotypes on the basis of various phenotypic and genetic traits, and by sensitivity to specific bacteriophages. Classical cholera tends to be clinically more

\section{Competing interests}

J. Clemens declares an association with the following company: Shantha Biotechnic. J. Holmgren declares an association with the following company: Crucell. See the article online for full details of the relationships. The other authors declare no competing interests. severe than El Tor cholera, but the El Tor biotype can survive better in the environment and is highly transmissible. ${ }^{7}$ These two biotypes are further differentiated into serotypes based on shared and distinct epitopes of the $\mathrm{O}$ antigen. The major serotypes are Inaba and Ogawa, but a third uncommon and usually unstable serotype (Hikojima) displays features of the other two types.

Although cholera outbreaks have been described since antiquity, seven pandemics have occurred since the early $19^{\text {th }}$ century, six of which arose in the Indian subcontinent. ${ }^{89}$ During the fifth pandemic, Robert Koch isolated the causative organism, which he termed the 'comma bacillus'. The sixth (and probably also the fifth) pandemics were caused by the classical biotype of $\mathrm{V}$. cholerae. The current (seventh) pandemic, caused by El Tor biotype cholera rather than the classical $V$. cholerae $\mathrm{O} 1$ biotype, arose in Sulawesi, Indonesia in 1961, and has subsequently spread to Southeast and South Asia, the Middle East and Europe, reaching sub-Saharan Africa in the 1970s. ${ }^{10-12}$ In 1991, the seventh pandemic reached South and Central America. ${ }^{13,14}$

Epidemic cholera was caused only by the O1 serogroup until 1992, when a major outbreak of disease caused by the $\mathrm{O} 139$ serogroup began in India ${ }^{15}$ and rapidly spread throughout Asia, but its incidence has now declined. ${ }^{16-18}$ Although researchers have speculated that this newly emergent organism might cause the eighth pandemic, this scenario has not yet materialized. ${ }^{19}$ Variants of $V$. cholerae $\mathrm{O} 1$, in which the organism is phenotypically similar to El Tor but encodes the cholera toxin expressed by the classical biotype, have also emerged in the past two decades. These were initially described in Bangladesh, and have subsequently been detected in India, Mozambique and numerous other countries in Asia and Africa, including
International Vaccine Institute, Seoul National University Research Park, San 4-8, Nakseongdaedong, Kwanak-gu, Seoul 151-919, Korea (J. Clemens, S. Shin) National Institute of Cholera and Enteric Diseases, P-33, CIT Scheme XM, Beliaghata, Kolkata 700 010, India (D. Sur, G. B. Nair). University of Goteborg, Box 435,

S-405 30 Gothenburg, Sweden (J. Holmgren).

Correspondence to:

J. Clemens

jclemens@ivi.int 


\section{REVIEWS}

\section{Key points}

- Early-generation cholera vaccines were delivered parenterally, but proved to have limited efficacy

- New understanding has yielded the insight that protective immunity is best generated by oral administration of cholera antigens

- Oral cholera vaccines comprising killed whole bacteria with or without cholera toxin B subunit have proved safe and effective but require at least two doses to achieve primary immunization

- One oral vaccine, containing killed whole cells and cholera toxin B subunit, is internationally licensed and WHO-prequalified

- Another oral, killed whole-cell cholera vaccine is licensed in India, can be produced inexpensively, and is likely to be WHO-prequalified for global use

- A third oral vaccine, consisting of live, genetically attenuated cholera organisms, has been licensed as a single dose vaccine but is currently not produced the order of $50 \%$ have been reported for severe cholera. Appropriate rehydration - the cornerstone of treatment-has lowered this rate to below $1 \%$. Nevertheless, severely dehydrating disease is an uncommon result of infection by $V$. cholerae. Most infections are asymptomatic or accompanied by mild diarrhea, and estimates of the ratio of symptomatic to asymptomatic infections range from 1:3 to 1:100. ${ }^{29}$ The numbers of cholera cases and deaths reported to official agencies seriously underestimates the true burden of this disease. The true global burden of cholera is thought to be on the order of 3 million cases and 100,000 deaths annually. Approximately half the burden of mortality is borne by children under 5 years old, and around half occurs in populations in sub-Saharan Africa (B. Maskery, personal communication).

Cholera occurs in endemic and epidemic epidemiological patterns. ${ }^{30}$ Although these represent extreme ends of a spectrum, the distinction is conceptually useful. Endemic cholera, as occurs in Bangladesh, is a disease that recurrently affects the same population and the source of infection is often marine reservoirs. ${ }^{31-37}$ Although environmental persistence of $V$. cholerae does not require contamination of these water sources by feces from infected humans, person-to-person transmission of cholera via fecal contamination has a very important role in the epidemiology of the disease in endemic settings. ${ }^{38,39}$ Owing to the recurrent pattern of endemic cholera, affected populations develop natural immunity that increases with age, and young children without previous exposure to the organism typically experience the highest incidence rates and the greatest clinical severity of disease. At the other end of the spectrum, epidemic cholera typically occurs unpredictably in a population that does not normally experience cholera, and the source of infection is often exogenous (such as a contaminated source of drinking water). Such populations lack pre-existing natural immunity, and consequently the incidence and severity of cholera in these settings tends to be age-independent..$^{30}$ The massive outbreak of cholera in Haiti in 2010 illustrates this pattern. ${ }^{3}$

Populations living in conditions with unclean water sources and poor sanitation are particularly at risk from cholera, and refugee settings seem to have a very high

RS2 region

b

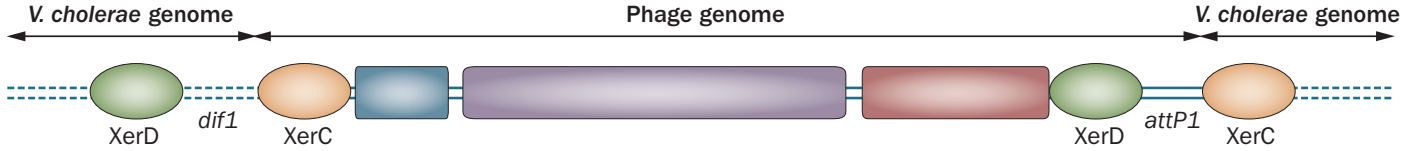

Figure 1 | Schematic representation of the CTX prophage. a | Cholera toxin is encoded by the CTX prophage in toxigenic Vibrio cholerae 01, and a typical CTX genome consists of a core region carrying the cholera toxin genes ctxAB, and psh, cep, pIIICTX, ace, zot—genes responsible for virion morphogenesis. The RS2 region encodes the regulation ( $r s t R$ ), replication $(r s t A)$, and integration ( $r s t B$ ) functions of the CTX genome. Direction of transcription is shown by the arrows. $\mathbf{b} \mid$ The integration process involves site-specific recombination, catalyzed by tryosine recombinases XerC and XerD, between the attP site (cataca) of СTXФ and the attB sequences inside the dif region (tgccgcgccaca) of chromosome 1 of $V$. cholerae. 
risk of cholera outbreaks. Additional patient-related risk factors include gastric hypochlorhydria, ${ }^{40-42}$ the lack of breastfeeding in infants ${ }^{43,44}$ and ABO blood group. ${ }^{45-50}$ Individuals with blood group $\mathrm{O}$ are at the highest risk of disease, followed by those with types A or B. Individuals with type $\mathrm{AB}$ are at the lowest risk of disease but, curiously, this relationship with blood type applies to the El Tor biotype and the O139 serogroup, but not to classical biotype cholera.

\section{Pathogenesis and immunology}

After ingestion, $V$. cholerae colonizes the small intestine, relying on several features of the organism including the toxin-coregulated pilus, as well as a hemagglutin, a protease, and the organism's single flagellum (Figure 2). ${ }^{51-55}$ Motility, made possible by the flagellum and the expression of strong mucolytic enzymes, such as mucinase, allows the organism to penetrate the intestinal mucosal lining. There, close association with the intestinal epithelium enhances binding of the cholera toxin to GM1 ganglioside receptors on epithelial cells. The toxin consists of a central enzymatic A subunit (consisting of $A 1$ and $A 2$ subunits) surrounded by a pentameric B subunit. ${ }^{56-58}$ The $B$ subunit binds to the GM1 ganglioside receptor, after which the A subunit is internalized into the cells through receptor-mediated endocytosis. Once internalized, the A subunit stimulates the enzyme adenylate cyclase, increasing levels of cyclic AMP and leading to the active secretion of chloride ions and inhibition of absorption of sodium chloride, which can cause prolific watery diarrhea (Figure 2). ${ }^{59}$

Immunity against cholera is thought to derive principally from the induction of mucosally secreted IgA antibodies directed against antibacterial antigens, primarily $V$. cholerae-derived lipopolysaccharide and cholera toxin. ${ }^{60,61}$ Antitoxin immunity (directed against the B subunit of cholera toxin), although appreciated to be of less importance than antibacterial immunity, seems to act synergistically with antibacterial immunity in protecting against cholera and is cross-reactive against the heat-labile toxin of enterotoxigenic Escherichia coli. ${ }^{62}$ Thus, the distinct functional roles of the A and B subunits of cholera toxin have relevance for the design of cholera vaccines, since the B subunit does not by itself induce secretion of fluid and electrolytes, although it is able to stimulate the production of antitoxin secretory IgA antibodies by the intestinal mucosa. This functional dissociation between immunogenicity and pathogenicity offered by the B subunit has led to its inclusion in several oral cholera vaccines. Natural immunity is not crossreactive between $\mathrm{O} 1$ and $\mathrm{O} 139$ serogroups. ${ }^{63,64}$ Thus, vaccines designed to protect against both $\mathrm{O} 1$ and $\mathrm{O} 139$ cholera serogroups, by eliciting anti-O antigen immunity, should contain both antigens. The existence of the Inaba and Ogawa serotypes also has implications for vaccine development, since in cholera-endemic settings, immune responses to Inaba infections are thought to protect against both Inaba and Ogawa serotypes, but the immunity induced by Ogawa seems to be mainly serotype-homologous. ${ }^{64-66}$

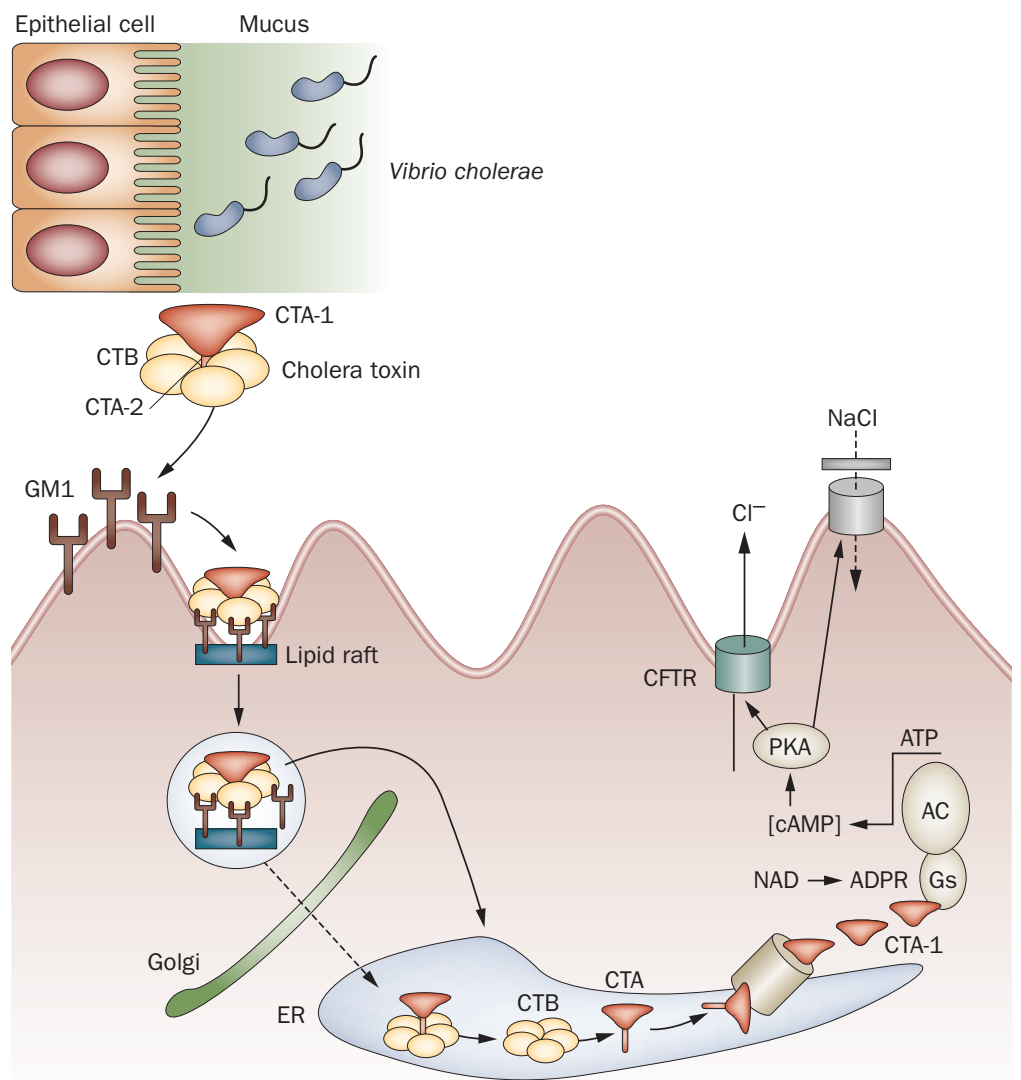

Figure 2 | Cholera pathogenesis and cholera toxin action. After ingestion, $V$. cholerae colonizes the small intestine and secretes cholera toxin, which has a doughnut-like structure with a central enzymatic toxic-active $A\left(A_{1}+A_{2}\right)$ subunit associated with pentameric $B$ subunits (B5). After binding to GM1 ganglioside receptors, mainly localized in lipid rafts on the cell surface, the toxin is endocytosed and travels to the ER via a retrograde pathway which-dependent on cell type-may or may not involve passage through the Golgi. In the ER, the A subunit dissociates from the $B$ subunits and through translocation via the ER degradasome pathway, $\mathrm{A} 1$ can reach the cytosol where it can rapidly refold. It binds to and ADP-ribosylates Gs, stimulating the AC complex to produce increased cellular levels of CAMP, leading to activation of PKA, phosphorylation of the major chloride channel, CFTR, and secretion of chloride $\left(\mathrm{Cl}^{-}\right)$and water. Cholera toxininduced chloride (and bicarbonate) secretion is especially pronounced from intestinal crypt cells, whilst in villus cells the increased cAMP levels instead mainly inhibits the normal uptake of $\mathrm{NaCl}$ and water ${ }^{14}$ Abbreviations: $\mathrm{AC}$, adenylate cyclase; ADPR, ADP ribose; CAMP, cyclic AMP; CTA, cholera toxin A; CTB, cholera toxin $\mathrm{B}$; CTFR, cystic fibrosis transmembrane conductance regulator; ER, endoplasmic reticulum; Gs, GTP- binding protein, Gs; PKA, protein kinase A.

\section{Cholera vaccines}

Injectable, killed whole-cell cholera vaccines were developed in the late $19^{\text {th }}$ century, not long after discovery of the causative organism. ${ }^{67}$ Early studies showed that they were protective, and these vaccines were widely used well into the $20^{\text {th }}$ century. ${ }^{67,68}$ However, properly designed clinical trials in East Pakistan (now Bangladesh), India, the Philippines, and Indonesia ultimately found that the vaccines usually conferred only modest protection (about 50\%) for less than 6 months. ${ }^{69,70}$ The vaccines were also associated with adverse effects such as fever, injection site pain, headache, and malaise. In conjunction with the recognition that prompt and adequate rehydration is a highly effective treatment for cholera, these findings led the WHO to abandon use of 
injectable cholera vaccines in public health programs in the 1970s. ${ }^{1,71}$

Several lines of evidence indicated that natural cholera infections are immunizing and suggested that an effective cholera vaccine should be possible. Human volunteers experimentally challenged with virulent $V$. cholerae $\mathrm{O} 1$ are protected against a second challenge for as long as 5 years after the first. ${ }^{72}$ Additionally, cohort studies of patients with endemic cholera have shown that symptomatic infections are associated with protection against recurrent disease, a phenomenon that seems to be serogroup-specific. ${ }^{64,73}$ These observations are explained by the fact that parenteral immunization is a relatively inefficient method of inducing protective secretory $\operatorname{IgA}$ antibodies in the intestine (and seems to require prior immunological priming) but oral administration of antigens can efficiently induce such antibodies. ${ }^{74}$

Attention consequently turned to the development of oral rather than parenteral cholera vaccines. ${ }^{75}$ The three currently licensed oral cholera vaccines, which contain either genetically attenuated live organisms or killed whole V. cholerae cells with or without additional antigens, are described below.

\section{Licensed new-generation oral cholera vaccines Dukoral $^{\circledR}$}

Dukoral $^{\circledR}$ (Crucell, Leiden, The Netherlands), was the first oral cholera vaccine to achieve international licensure. ${ }^{76}$ This vaccine contains formalin-killed or heat-killed whole cells of the O1 serogroup (a mixture of classical and El Tor biotypes, including both Ogawa and Inaba serotypes) together with recombinant cholera toxin B subunit. This vaccine must be coadministered with a bicarbonate buffer to prevent disaggregation of the pentameric B subunit in the acidic gastric environment. ${ }^{77}$

Use of this combination of constituents was predicated on the notion that inducing both antibacterial and antitoxic immunity would confer synergistic protection against cholera. ${ }^{74,78}$ The vaccine was originally produced in the 1980s as a B subunit-whole-cell vaccine, in which the cholera toxin B subunit was obtained via chemical extraction. The efficacy of this vaccine was demonstrated by a 1985 field trial in Bangladesh, in which 89,596 persons aged 2-15 years and women over the age of 15 years were randomly allocated to receive three doses of the B subunit-whole-cell vaccine, a vaccine containing the killed whole-cell constituents only, or placebo. The whole-cell-only vaccine had a protective efficacy of about $50 \%$, which was sustained for 3 years. ${ }^{79-83}$ The B subunit-whole-cell vaccine conferred the best shortterm protective effects: the efficacy of this vaccine was $85 \%$ in the first $4-6$ months, $62 \%$ during the first year, $57 \%$ during the second year, and negligible thereafter. No adverse effects could be attributed to vaccination. Several additional findings on protection by this B subunitwhole-cell vaccine have been reported. The protection achieved by two doses seemed to be equivalent to that offered by three doses, and the vaccine provided greater defense against classical than El Tor cholera. People vaccinated after 5 years of age were better protected than those receiving the vaccine between the ages of 2 and 5 years. Short-term cross-protection was observed against diarrhea caused by heat labile toxin-producing enterotoxigenic E. coli. ${ }^{62}$

A two-dose regimen of another version of this vaccine, containing cholera toxin B subunit produced by recombinant genetic technology plus the killed whole-cell constituents described above, was subsequently evaluated in two placebo-controlled, randomized trials conducted in Peru. ${ }^{84,85}$ In the first, 1,426 Peruvian soldiers were randomly allocated to receive a two-dose regimen of recombinant B subunit-whole-cell vaccine or placebo. During an outbreak of cholera that occurred several months later, the vaccine efficacy was $86 \%$, a value nearly identical to that for short-term protection with the chemically extracted B subunit-whole-cell vaccine in Bangladesh. ${ }^{84}$ In the second trial, 17,799 individuals aged 2-65 years were randomly allocated to receive a two-dose regimen of recombinant B subunit-whole-cell vaccine or placebo. No protection was observed after completion of this primary regimen, but after a third (booster) dose of the same vaccine 10 months later, vaccine protective efficacy was $61 \% .{ }^{85}$ Methodological differences to the previously published studies might explain this apparent lack of efficacy. ${ }^{86}$

On the basis of data from these phase III trials, the recombinant B subunit-whole-cell oral vaccine was licensed in Europe and subsequently elsewhere, as a two-dose regimen given with a buffer to people aged over 5 years, with repeat dosing 2 years later; and as a three-dose regimen for children $2-5$ years of age, with a booster dose 6 months later. For each age group, the doses for the primary regimen are given 1-6 weeks apart (Table 1). Postlicensure studies showed that this vaccine was safe in HIV-infected volunteers. ${ }^{87,88}$

Another study in Beira, Mozambique-a city with a high prevalence of HIV infections-found that mass immunization of males and nonpregnant females aged $\geq 2$ years with a two-dose regimen was associated with $78 \%$ protection against episodes of cholera detected in treatment centers, and $89 \%$ protection against those episodes associated with severe dehydration during 1 year of follow-up. ${ }^{89}$ This finding is of particular interest, given that all cholera isolates from patients in this study were the newly emergent variant El Tor strains that produce classical cholera toxin. ${ }^{90}$

\section{ORCVAX ${ }^{\circledR}$, mORCVAX $^{\circledR}$ and Shancho ${ }^{\circledR}$}

Motivated by the positive findings from the 1985 trial in Bangladesh, the Vietnamese government implemented local development of an inexpensive oral whole-cell-only vaccine, in the late 1980s. The two-dose, O1-serogrouponly, killed whole-cell vaccine, similar in composition to $\mathrm{Dukoral}^{\circledast}$, was safe and conferred $66 \%$ protection against cholera at 8-10 months following vaccination in an open field trial in Hue, Vietnam, in the early 1990s. ${ }^{91}$ This vaccine, licensed as ORCVAX ${ }^{\circledR}$ (Vabiotech, Hanoi, Vietnam) in 1997, had the additional advantage of not requiring coadministration of oral buffer.

A postlicensure study of a two-dose regimen of ORCVAX $^{\circledR}$ found that mass immunization in the city 
Table 1 | Licensed oral cholera vaccines*

\begin{tabular}{|c|c|c|c|}
\hline Characteristics & Dukoral $^{\circledR}$ & Shanchol $^{\circledR}$, mORCVAX $^{\circledR}$ & Orochol $^{\circledR}$, Mutachol ${ }^{\circledR}$ \\
\hline Constituents & $\begin{array}{l}\text { Killed whole cells ( } 01 \text { classical and } \\
\text { El Tor biotypes) plus recombinant } \\
\text { B subunit }\end{array}$ & $\begin{array}{l}\text { Killed whole cells only (01 classical } \\
\text { and El Tor biotypes plus } 0139 \text { ) }\end{array}$ & $\begin{array}{l}\text { Live genetically attenuated cells } \\
\text { (CVD 103-HgR } 01 \text { classical) }\end{array}$ \\
\hline Regimen & $\begin{array}{l}2 \text { doses given } 7-42 \text { days apart } \\
\text { ( } 3 \text { doses for children aged } 2-5 \text { years) }\end{array}$ & 2 doses given 14 days apart & 1 dose \\
\hline $\begin{array}{l}\text { Duration of } \\
\text { protection }\end{array}$ & $\begin{array}{l}2 \text { years ( } 6 \text { months for children aged } \\
2-5 \text { years) }\end{array}$ & 3 years & $\begin{array}{l}6 \text { months (established only } \\
\text { in North American volunteers) }\end{array}$ \\
\hline $\begin{array}{l}\text { Booster dose } \\
\text { requirements }\end{array}$ & $\begin{array}{l}\text { Every } 2 \text { years (every } 6 \text { months for } \\
\text { children aged } 2-5 \text { years) }\end{array}$ & $\begin{array}{l}\text { Every } 2 \text { years (likely to be extended } \\
\text { to every } 3 \text { years) }\end{array}$ & Unknown \\
\hline $\begin{array}{l}\text { Age range for } \\
\text { vaccination }\end{array}$ & $\geq 2$ years & $\begin{array}{l}\text { Shanchol}^{\oplus} \geq 1 \text { year; mORCVAX } \\
\geq 2 \text { years }\end{array}$ & $\geq 2$ years \\
\hline $\begin{array}{l}\text { Requirement } \\
\text { for oral buffer }\end{array}$ & Yes & No & Yes \\
\hline Shelf life & 3 years & 2 years & 2 years \\
\hline $\begin{array}{l}\text { International } \\
\text { acceptance }\end{array}$ & WHO prequalified & Pending WHO prequalification & Not prequalified by WHO \\
\hline
\end{tabular}

of Hue was associated with a $50 \%$ protection against symptomatic cholera for at least 3 years after vaccination..$^{93}$ Another study of this vaccine during the $2007-$ 2008 cholera epidemic in Hanoi demonstrated 76\% protection in the 3-5 months following vaccination. ${ }^{93}$

Over 20 million doses of ORCVAX ${ }^{\circledR}$ were used in Vietnam's public health programs. ${ }^{94,95}$ However, ORCVAX $^{\circledR}$ (which was subsequently made bivalent, containing both $\mathrm{O} 1$ and $\mathrm{O} 139$ serogroups) was not suitable for international use because of several production and standardization problems, and because the Vietnamese national regulatory authority was not WHO-approved. ${ }^{97}$ Accordingly, in 2004, the International Vaccine Institute in Seoul, Korea, initiated a program to develop an improved version of this inexpensive and easily produced vaccine suitable for international use, with optimized production technology, quality control procedures and standardization assays.

Production of the modified bivalent vaccine was transferred to Shantha Biotechnics in India, whose national regulatory authority is WHO-approved. Subsequently, Vietnam has begun to produce this modified vaccine, which is now used in Vietnam's public health programs. A two-dose regimen of the modified $\mathrm{O} 1$ and O139 serogroup, killed whole-cell vaccine was safe and elicited serum anti-O1 vibriocidal antibodies (the standard measure of immune responses used in evaluations of cholera vaccines) in phase II trials carried out in Vietnamese adults, and in both adults and children in Kolkata, India. ${ }^{97,98}$ Titers of these antibodies were several times higher than those observed in the early studies of $\mathrm{ORCVAX}^{\circledR}$, although the serum levels of anti-O139 vibriocidal antibodies were modest. ${ }^{99,100}$ A large, randomized, placebo-controlled trial of this vaccine in 69,328 persons aged 1 year and older in Kolkata demonstrated $66 \%$ protection against $\mathrm{O} 1$ serogroup cholera, with no decline in protection during 3 years of follow-up. ${ }^{101,102}$ All episodes of cholera in this trial were caused by the newly emergent El Tor biotype that produces classical biotype cholera toxin.

The modified vaccine was licensed in India as Shanchol ${ }^{\circledR}$ (Shantha Biotechnics, Hyderabad, India) in 2009 and as mORCVAX ${ }^{\circledR}$ (VaBiotech, Hanoi, Vietnam) in Vietnam in 2010. The vaccine is administered as a twodose regimen for persons aged 1 year and older, and does not require an oral buffer. At present, repeat vaccination is recommended every 2 years, but data obtained after 3 years of follow-up suggest that this interval will lengthen to at least 3 years (Table 1). ${ }^{102}$

\section{Orochol ${ }^{\circledR}$ and Mutachol ${ }^{\circledR}$}

To date, only one genetically attenuated, live oral cholera vaccine has achieved licensure. This vaccine is derived from the virulent $\mathrm{O} 1$ serogroup, classical Inaba strain 569B. The basis for its attenuation is deletion of the gene encoding cholera toxin A subunit, while still expressing cholera toxin B subunit. The strain was further engineered to be mercury-resistant, which serves as a marker capable of distinguishing the vaccine organism (termed CVD 103-HgR) from wild-type strains. ${ }^{103}$ This vaccine, which is given as a single dose, was tested in phase I-II studies that enrolled over 4,000 volunteers and was safe at doses of up to $5 \times 10^{9}$ viable organisms. ${ }^{104-108}$ Doses of $2-8 \times 10^{8}$ viable organisms were reliably immunogenic and protective against experimental challenge with both Inaba and Ogawa cholera serotypes in North American volunteers; ${ }^{109}$ and protection was seen as early as 1 week and as late as 24 weeks after dosing. However, when tested in developing countries, serum vibriocidal antibody responses to a $1 \times 10^{8}$ vaccine dose were substantially lower in magnitude than those seen in North American volunteers. ${ }^{110}$

Accordingly, when this attenuated live vaccine entered phase III testing for efficacy in an endemic cholera setting, the dose selected was $5 \times 10^{9}$ organisms. In this trial, carried out between 1993 and 1997 in North 

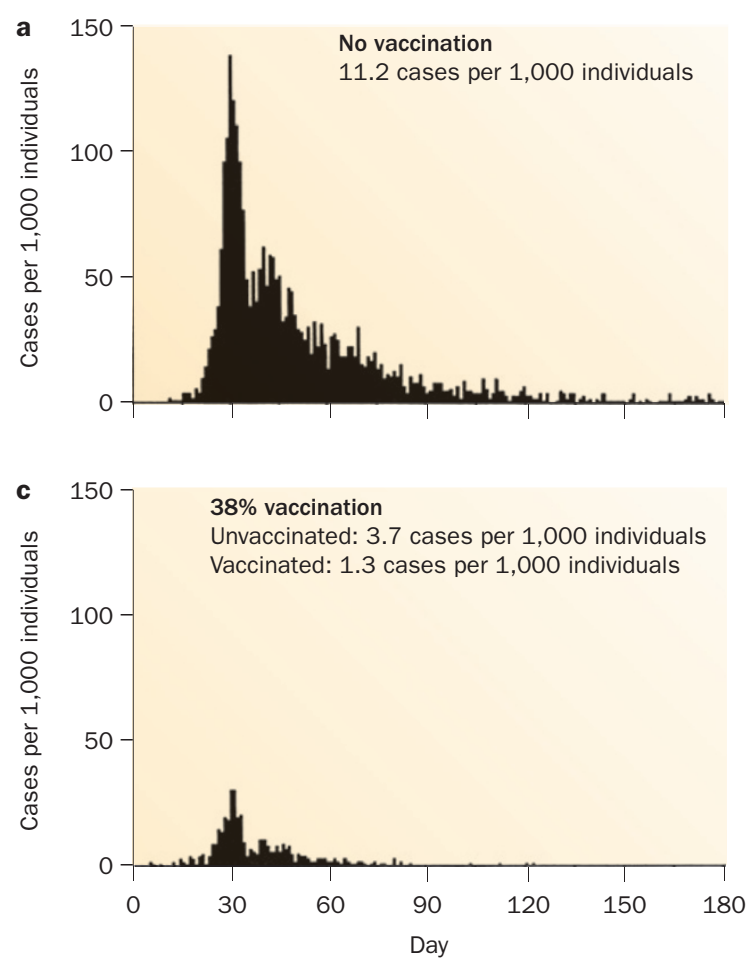

b

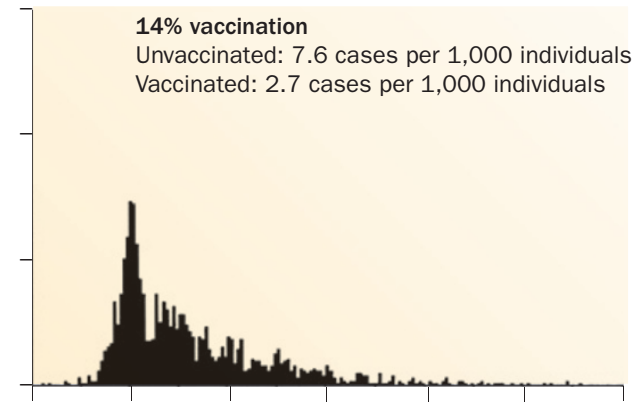

d

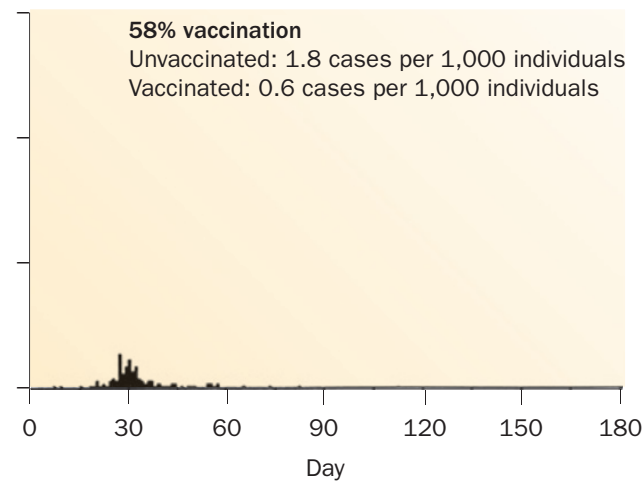

Figure 3 | Caseload of cholera in rural Bangladesh predicted by a dynamic transmission mathematical model.

a | Anticipated caseload of cholera during a year following no vaccination and $\mathbf{b}-\mathbf{d} \mid$ following vaccination with progressively higher levels of vaccine coverage. Cholera is predicted to be nearly extinguished by a vaccine coverage level of only around 60\%. Adapted from Longini I. M. Jr et al. PLoS Med. 4, e336 (2007), which is published under an Creative Commons Attribution License by Public Library of Science.

Jakarta, Indonesia, 67,508 persons aged 2-41 years were randomly allocated to receive either a single dose of the genetically attenuated organism or placebo. ${ }^{111}$ Vaccine efficacy against symptomatic (treated) episodes of $\mathrm{O} 1$ serogroup cholera was $14 \%$ at 4 years of follow-up; no significant protection was observed during any year of follow-up, though a paucity of cases in the first 6 months precluded assessment of short-term efficacy. ${ }^{111}$

CVD103-HgR was licensed in 1993 as Orochol $^{\circledR}$ and as Mutachol ${ }^{\circledast}$ (Crucell, Bern, Switzerland) at a dose of $2 \times 10^{8}$ viable organisms for travelers aged 2 years and older. A dose of $2 \times 10^{9}$ is licensed as Orochol E ${ }^{\circledR}$ (Crucell), which is intended for use in developing countries, but to date this product has not been used in the routine public health programs of any such countries. Orochol ${ }^{\circledR}$, Orochol $\mathrm{E}^{\circledR}$ and Mutachol $^{\circledR}$ are given as a single dose with a booster dose recommended 6 months later (Table 1). The vaccine is coadministered with oral buffer, as the vaccine organism is acid-sensitive. However, at present these vaccines are not being manufactured.

A postlicensure study of mass immunization with a single dose of $2 \times 10^{9}$ viable CVD103-HgR organisms, given following the onset of an epidemic in Micronesia, found that vaccination was associated with a $79 \%$ reduction in the risk of cholera. ${ }^{112}$ However, because this was not a double-blinded, randomized, controlled trial and because the results were at variance with those of the trial in North Jakarta, the findings will require confirmation in future studies. Another postlicensure study with a similar dose found the vaccine to be safe, albeit associated with reduced immune responses, in HIV-infected adults in Mali. ${ }^{113}$

\section{Considerations for use of current vaccines}

$V$. cholerae continues to evolve, and consequently the observation that currently licensed killed oral cholera vaccines also protect against cholera caused by the newly emergent El Tor variants that produce classical biotype cholera toxin is reassuring. ${ }^{89,101}$ An important insight from the phase III trial of killed oral cholera vaccines in Bangladesh was that these vaccines not only achieve direct individual protection for vaccinated individuals, but also result in herd immunity. ${ }^{114,115}$ For example, mathematical models predict that Dukoral ${ }^{\circledR}$ vaccination of only around $60 \%$ of the population would be adequate to almost extinguish the annual caseload of cholera in rural Bangladesh, where the disease is hyperendemic (Figure 3). ${ }^{116}$

Effective induction of herd immunity greatly improves the cost-effectiveness of killed oral cholera vaccines. A study of an oral killed whole-cell vaccine with the features of Shanchol ${ }^{\circledR}$ used empiric data from several sites in Asia and Africa on cholera incidence and the costs associated with three different vaccination programs to evaluate the cost-effectiveness of vaccination, taking into account both direct protective effects and herd immunity. ${ }^{117}$ The researchers found in Kolkata, India and Beira, Mozambique that vaccination programs targeting three 
different age ranges (school-aged children [5-14 years], children aged 1-14 years, and all persons aged 1 year and over) were very cost-effective, according to WHO criteria, when vaccine herd immunity and direct protective effects were both taken into account. By contrast, when vaccine herd immunity was not considered, vaccination was only cost-effective in both sites when children aged 1-14 years were targeted. ${ }^{117}$

Vaccination with killed oral cholera vaccines has been shown to be feasible in endemic areas, refugee settings and humanitarian emergencies, including those in Beira, Mozambique; Hue, Vietnam; Darfur, Sudan; Udjumani, Uganda; and Aceh, Indonesia. ${ }^{118-120}$ Reactive vaccination with oral cholera vaccines was also demonstrated to be feasible after the onset of epidemics that occurred in Vietnam and Micronesia. ${ }^{93,112}$ Models show that if killed oral cholera vaccines had been promptly and reactively deployed during recent cholera outbreaks in Haiti and Zimbabwe, they would have had a major protective effect, due partly to the prolonged durations of these outbreaks-a feature shared with several other recent outbreaks. ${ }^{121,122}$

Currently, WHO recommends the use of oral cholera vaccines for populations at definably high risk of endemic cholera. In addition, WHO recommends that reactive deployment of these vaccines should be considered in cholera epidemics-if epidemiological and logistical circumstances are judged to be favorable for vaccine use. ${ }^{123}$ With the availability of one WHO-prequalified vaccine, Dukoral $^{\circledR}$, and the imminent availability of another, Shanchol ${ }^{\circledast}$, we anticipate that oral cholera vaccines will increasingly be used in public health programs in the years to come.

\section{Potential future vaccine candidates}

Several other live oral vaccines against O1 serogroup cholera are currently in development. The most advanced of these is Peru-15, which was genetically engineered from an El Tor Inaba wild-type strain recovered in Peru. In this vaccine strain, the entire cholera toxin cassette has been deleted, including the flanking recombination sites, and a gene for cholera toxin B subunit has been inserted instead. A single-dose regimen of $1 \times 10^{8}$ viable vaccine organisms was safe, as well as protective against experimental cholera challenges in American volunteers, and immunogenic in Bangladeshi adults, children, and infants. ${ }^{124-127}$

Other live oral vaccine candidates include a Cuban strain (638), developed by genetic engineering of an El Tor Ogawa strain; ${ }^{128,129}$ another El Tor Ogawa strain developed in China (IEM108), which is naturally deficient in the cholera toxin prophage and has been further engineered to include the cholera toxin B subunit and $r s t R$ genes; ${ }^{130,131}$ and a strain developed in India (VA1.3), also lacking the cholera toxin prophage, and into which the gene for cholera toxin B subunit has been inserted. ${ }^{132}$ Each is given as a single dose, although only the 638 and VA1.3 strains have been tested in humans.

Attention has also focused on developing live O139 serogroup vaccine strains. Two single-dose oral vaccine candidates have reached human testing: Bengal 15 was genetically constructed using the same strategy as for Peru $15,{ }^{133}$ whereas CVD 112 was genetically constructed using a similar approach to that used to develop CVD103-HgR. ${ }^{134}$ Single-dose regimens of both candidates were safe and protected against serogroup O139 challenges in North American adults. A third, live O139 serogroup vaccine candidate developed in Cuba is based on a strain in which the cholera toxin prophage has been spontaneously deleted; additional gene deletions were then made. ${ }^{135}$ This vaccine has yet to be tested in humans.

Finally, investigators at the NIH have developed parenterally administered vaccine candidates based on detoxified lipopolysaccharide from $V$. cholerae conjugated to a carrier protein. Conjugates in which lipopolysaccharide from the classical Inaba (569B) strain was conjugated to cholera toxin elicited titers of vibriocidal antibodies in the sera of human volunteers that were higher than those observed after parenteral administration of whole-cell cholera vaccine. ${ }^{136}$ Similar approaches have been used to create O139 serogroup conjugates, which have demonstrated immunogenicity and protection in animal studies. ${ }^{137,138}$

\section{Conclusions}

In over 100 years of cholera vaccine development and use, we have passed from widespread use of parenteral vaccines that proved to be inadequately protective in well-designed clinical trials, through to rejection of vaccination as a strategy for control of cholera. Now, with the development and availability of safe and effective oral vaccines, we enter an era in which vaccines are expected to find a role in the public health armamentarium to control cholera.

The rationale for using these vaccines in programs to control endemic cholera in populations that are at definably high risk is now widely accepted. Reactive use of vaccines in the control of cholera epidemics is still in debate. Creation of a global cholera vaccine stockpile for use during such epidemics would provide a mechanism not only for timely vaccine deployment, but also for research to refine strategies for vaccine use. ${ }^{139}$ In the meantime, work on development of improved vaccines continues. Of special interest are the live oral vaccine candidates that could be administered as a single dose. The availability of a single-dose vaccine would greatly simplify use of vaccines in the control of both endemic and epidemic cholera.

\footnotetext{
Review criteria

We searched PubMed for original articles in the English language published in 1960-2011 that focused on cholera vaccines and vaccination strategies. The search terms used were "Vibrio cholerae", "cholera", and "cholera vaccines". We also included additional papers identified by searching the reference lists of selected articles identified in the PubMed search, as well as other manuscripts and book chapters known to be pertinent.
} 
1. Sack, D. A., Sack, R. B., Nair, G. B. \& Siddique, A. K. Cholera. Lancet 363, 223-233 (2004).

2. World Health Organization. Cholera. Weekly Epidemiological Record. 85, 293-308 (2010).

3. Harris, J. B. et al. Cholera's western front. Lancet 376, 1961-1965 (2010).

4. Mintz, E. \& Guerrant, R. A lion in our village-the unconscionable tragedy of cholera in Africa. N. Engl. J. Med. 360, 1060-1063 (2009).

5. Glass, R. I. et al. Endemic cholera in Bangladesh, 1966-1980. Am. J. Epidemiol. 116, 959-970 (1982).

6. McCleod, S. M., Kimsey, H. H., Davis, B. M. \& Waldor, M. K. CTX $\varphi$ and Vibrio cholerae: exploring a newly recognized type of phage-host cell relationship. Mol. Microbiol. 57, 347-366 (2005).

7. Woodward, W. \& Mosley, W. H. The spectrum of cholera in rural Bangladesh. II. Comparison of El Tor Ogawa and classical Inaba infections. J. Infect. Dis. 96, 342-351 (1972).

8. Pollitzer, R. Cholera. Geneva. World Health Organization. (1959).

9. Barua, D. in Vibrio cholerae and Cholera (ed. Takeda, Y.) 9-32 (KTK Scientific Publishers, Tokyo, 1988).

10. Felix, $\mathrm{H}$. The development of the cholera epidemic in West Africa. Bull. Soc. Pathol. Exot Filiales. 64, 561-582 (1971).

11. Goodgame, R. \& Greenough, W. Cholera in Africa. Ann. Intern. Med. 82, 101-106 (1975).

12. Blake, P. A. et al. Cholera in Portugal, 1974. I. Modes of transmission. Am. J. Epidemiol. 105, 337-343 (1977).

13. Quick, R. E. et al. Epidemic cholera in the Amazon: the challenge of preventing death. Am.J. Trop. Med. Hyg. 93, 597-602 (1993).

14. Swerdlow, D. L. et al. Waterborne transmission of epidemic cholera in Trujillo, Peru: lessons for a continent at risk. Lancet 340, 28-32 (1992).

15. Bhattacharya, M. K. et al. Outbreak of Vibrio cholerae non-01 in India and Bangladesh. Lancet 341, 1346-1347 (1993).

16. Garg, S. et al. Nationwide prevalence of the new epidemic strain of Vibrio cholerae 0139 Bengal in India. J. Infect. Dis. 27, 108-109 (1993).

17. Cholera Working Group. Large epidemic of cholera-like disease in Bangladesh caused by Vibrio cholerae 0139 synonym Bengal. Lancet 342, 387-390 (1992).

18. Morris, J. G. et al. Clinical and immunologic characteristics of Vibrio cholerae 0139 Bengal infection in North American volunteers. J. Infect. Dis. 171, 903-908 (1995).

19. Nair, G. B., Bhattacharya, S. \& Deb, B. Vibrio cholerae 0139 Bengal: the eighth pandemic strain of cholera. Indian J. Public Health 38 33-36 (1994).

20. Nair, G. B. et al. New variants of Vibrio cholerae 01 biotype El Tor with attributes of the classical biotype from hospitalized patients with acute diarrhea in Bangladesh. J. Clin. Microbiol. 40, 3296-3299 (2002).

21. Ansaruzzaman, M. et al. The Mozambique Cholera Vaccine Demonstration. Project Coordination Group. Cholera in Mozambique, Variant of Vibrio cholerae. Emerg. Infect. Dis. 10, 2057-2059 (2004)

22. Nair, G. B. et al. Cholera due to altered El Tor strains of Vibrio cholerae 01 in Bangladesh. J. Clin. Microbiol. 23, 4211-4213 (2006).

23. Taychoudhuri, A. et al. Classical ctxB in Vibrio cholerae 01, Kolkata, India. Emerg. Infect. Dis. 15, 131-132 (2009).

24. Safa, A., Sultana, J., Dac Cam, P., Mwansa, J. C. \& Kong, R. Y. Vibrio cholerae 01 hybrid EI Tor strains, Asia and Africa. Emerg. Infect. Dis. 14, 987-988 (2008).
25. Chin, C. S. et al. The origin of Haitian cholera outbreak strain. N. Engl. J. Med. 364, 33-42 (2011).

26. Siddique, A. et al. El Tor cholera with severe disease: a new threat to Asia and beyond. Epidemiol. Infect. 138, 347-352 (2010).

27. Carpenter, C. C. Cholera: diagnosis and treatment. Bull. NY Acad. Med. 47, 1192-1203 (1971).

28. Butler, T., Arnold, M. \& Islam, M. Depletion of hepatic glycogen in the hypoglycaemia of fatal childhood diarrhoeal illnesses. Trans. R. Soc. Trop. Med. Hyg. 83, 839-843 (1989).

29. Khan, M. \& Shahidullah, M. Cholera due to the E1 Tor biotype equals the classical biotype in severity and attack rates. J. Trop. Med. Hyg. 83, 35-39 (1980)

30. Clemens, J., Spriggs, D. \& Sack, D. in Vibrio cholerae and Cholera: Molecular to Global Perspectives (ed. Wachsmith, I. K., Blake, P. \& Olsvik, O.) 425-440 (American Society for Microbiology, Washington, 1994).

31. Blake, P. A. et al. Cholera-a possible endemic focus in the United States. N. Engl. J. Med. 302 , 305-309 (1980).

32. Nalin, D. R. Cholera, copepods, and chitinase. Lancet 2, 958 (1976).

33. Huq, A. et al. Colonization of the gut of the blue crab (Callinectes sapidus) by Vibrio cholerae. Appl. Environ. Microbiol. 52, 586-588 (1986).

34. Epstein, P. R. Algal blooms in the spread and persistence of cholera. Biosystems 31, 209-221 (1993).

35. Islam, M. S., Drasar, B. S. \& Bradley, D. J. Attachment of toxigenic Vibrio cholerae 01 to various freshwater plants and survival with a filamentous green alga, Rhizoclonium fontanum. J. Trop. Med. Hyg. 92, 396-401 (1989).

36. Huq, A. et al. Ecological relationships between Vibrio cholerae and planktonic crustacean copepods. Appl. Environ. Microbiol. 45 275-283 (1983).

37. Huq, A., West, P. A., Small, E. B., Huq, M. I. \& Colwell, R. R. Influence of water temperature, salinity, and $\mathrm{pH}$ on survival and growth of toxigenic Vibrio cholerae serovar 01 associated with live copepods in laboratory microcosms. Appl. Environ. Microbiol. 48, 420-424 (1984).

38. Merrell, D. S. et al. Host-induced epidemic spread of the cholera bacterium. Nature 417 , 642-645 (2002).

39. Alam, A. et al. Hyperinfectivity of humanpassaged Vibrio cholerae can be modeled by growth in the infant mouse. Infect. Immun. 73 6674-6679 (2005)

40. Nalin, D. R. et al. Cannabis, hypochlorhydria, and cholera. Lancet 2, 859-862 (1978)

41. Sack, G. H. Jr et al. Gastric acidity in cholera and noncholera diarrhoea. Bull. World Health Organ. 47, 31-36 (1972).

42. Merrell, D. S. \& Camilli, A. Acid tolerance of gastrointestinal pathogens. Curr. Opin. Microbiol. 5, 51-55 (2002).

43. Clemens, J. D. et al. Breast feeding and the risk of severe cholera in rural Bangladeshi children. Am. J. Epidemiol. 131, 400-411 (1990).

44. Glass, R. I. et al. Protection against cholera in breast-fed children by antibodies in breast milk. N. Engl. J. Med. 308, 1389-1392 (1983).

45. Sircar, B. K. et al. ABO blood group distributions in diarrhoea cases including cholera in Calcutta. Ann. Hum. Biol. 8, 289-291 (1981).

46. Black, R. E., Levine, M. M., Clements, M. L., Hughes, T. \& O'Donnell, S. Association between $\mathrm{O}$ blood group and occurrence and severity of diarrhoea due to Escherichia coli. Trans. R. Soc. Trop. Med. Hyg. 81, 120-123 (1987).
47. Clemens, J. D. et al. ABO blood groups and cholera: new observations on specificity of risk and modification of vaccine efficacy. J. Infect. Dis. 159, 770-773 (1989).

48. Holmgren, J., Lindblad, M., Fredman, P., Svennerholm, L. \& Myrvold, H. Comparison of receptors for cholera and Escherichia coli enterotoxins in human intestine. Gastroenterology 89, 27-35 (1985).

49. Glass, R. I. et al. Predisposition for cholera of individuals with $\mathrm{O}$ blood group: possible evolutionary significance. Am. J. Epidemiol. 121, 791-796 (1985).

50. Harris, J. B. et al. Blood group, immunity, and risk of infection with Vibrio cholerae in an area of endemicity. Infect. Immun. 73, 7422-7427 (2005).

51. Tacket, C. O. et al. Investigation of the roles of toxin-coregulated pili and mannose-sensitive hemagglutinin pili in the pathogenesis of Vibrio cholerae 0139 infection. Infect. Immun. 66 692-695 (1998).

52. Taylor, R. K., Miller, V. L., Furlong, D. B. \& Mekalanos, J. J. Use of phoA gene fusions to identify a pilus colonization factor coordinately regulated with cholera toxin. Proc. Natl Acad. Sci. USA 84, 2833-2837 (1987).

53. Silva, A. J., Leitch, G. J., Camilli, A. \& Benitez, J. A. Contribution of hemeagglutinin/ protease and motility to pathogenesis of El Tor biotype cholera. Infect. Immun. 74, 2072-2079 (2006)

54. Butler, S. \& Camilli, A. Going against the grain: chemotaxis and infection in Vibrio cholerae. Nat Rev. Microbiol. 3, 611-620 (2005).

55. Lee, S. H., Hava, D. L., Waldor, M. K. \& Camilli, A. Regulation and temporal expression patterns of Vibrio cholerae virulence genes during infection. Cell 99, 625-634 (1999).

56. Holmgren, J., Lonnroth, I. \& Ouchterlony, O. Identification and characterization of cholera exotoxin in culture filtrates of $V$. cholerae. Acta Pathol. Microbiol Scand. [B] Microbiol. Immunol. 79, 448 (1971).

57. Lonnroth, I. \& Holmgren, J. Subunit structure of cholera toxin. J. Gen. Microbiol. 76, 417-427 (1973).

58. Holmgren, J., Lonnroth, I. \& Svennerholm, L. Fixation and inactivation of cholera toxin by GM1 ganglioside. Scand. J. Infect. Dis. 5, 77-78 (1973)

59. Kimberg, D. V., Field, M., Johnson, J., Henderson, A. \& Gershon, E. Stimulation of intestinal mucosal adenyl cyclase by cholera enterotoxin and prostaglandins. J. Clin. Invest. 50, 1218-1230 (1971).

60. Holmgren, J. \& Svennerholm, A. M. Mechanisms of disease and immunity in cholera: a review. J. Infect. Dis. 136 (Suppl.), S105-S112 (1977).

61. Holmgren, J. \& Svennerholm, A. M. Cholera and the immune response. Prog. Allergy 22 106-119 (1983)

62. Clemens, J. et al. Cross-protection by B subunitkilled whole cell cholera vaccine against diarrhea associated with heat-labile toxinproducing enterotoxigenic Escherichia coli: results of a large-scale field trial. J. Infect. Dis. 158, 372-377 (1988).

63. Albert, M. J., Alam, K., Rahman, A. S., Huda, S. \& Sack, R. B. Lack of cross-protection against diarrhea due to Vibrio cholerae 01 after oral immunization of rabbits with, V. cholerae 0139 Bengal. J. Infect. Dis. 169, 709-710 (1994).

64. Ali, M., Emch, M., Park, J. K., Yunus, M. \& Clemens, J. Natural cholera infection-derived immunity in an endemic setting. J. Infect. Dis. 204, 912-918 (2011). 
65. Mosley, W. H., Aziz, K. M., Rahman, A. S., Chowdhury, A. K. \& Ahmed, A. Field trials of monovalent Ogawa and Inaba cholera vaccines in rural Bangladesh-three years of observation. Bull. World Health Organ. 49, 381-387 (1973)

66. Longini, I. M. Jr et al. Epidemic and endemic cholera trends over a 33-year period in Bangladesh. J. Infect. Dis. 186, 246-251 (2002).

67. Bornside, G. H. Waldemar Haffkine's cholera vaccines and the Ferran-Haffkine priority dispute. J. Hist. Med. Allied Sci. 37, 399-422 (1982).

68. Chandrasekar, C. Statistical assessment of the efficacy of anti-cholera inoculation from the data of 63 cheris in south Sercot district. Indian J. Med. Res. 35, 153 (1947).

69. Joo, I. Cholera (eds Barua, D. \& Burrows, W.) 333-349 (Saunders, Philadelphia, 1974).

70. Benenson, A. S., Joseph, P. R. \& Oseasohn, R. O. Cholera vaccine field trials in east Pakistan. 1 Reaction and antigenicity studies. Bull. World Health Organ. 38, 347-357 (1968).

71. World Health Organization and Global Task Force on Cholera Control. Guidelines for Cholera Control. Geneva: World Health Organization. (1994).

72. Cash, R. A. et al. Response of man to infection with Vibrio cholerae. II. Protection from illness afforded by previous disease and vaccine. J. Infect. Dis. 130, 325-333 (1974).

73. Clemens, J. D. et al. Biotype as determinant of natural immunising effect of cholera. Lancet 337, 883-884 (1991)

74. Svennerholm, A. M. et al. Mucosal antitoxic and antibacterial immunity after cholera disease and after immunization with a combined $B$ subunit-whole cell vaccine. J. Infect. Dis. 149 884-893 (1984)

75. Shin, S., Desai, S., Sah, B. K. \& Clemens, J. Oral vaccines against cholera. Clin. Infect. Dis. 52, 1343-1349 (2011).

76. Holmgren, J. et al. An oral B subunit: whole cell vaccine against cholera. Vaccine 10, 911-914 (1992).

77. Clemens, J. et al. Effect of neutralization of gastric acid on immune responses to oral B subunit killed whole cell cholera vaccine. J. Infect. Dis. 154, 175-178 (1986).

78. Svennerholm, A. M. \& Holmgren, J. Synergistic protective effect in rabbits of immunization with Vibrio cholerae lipopolysaccharide and toxin/ toxoid. Infect. Immun. 13, 735-740 (1976).

79. Clemens, J. et al. Field trial of oral cholera vaccines in Bangladesh: serum vibriocidal and antitoxic antibodies as markers of the risk of cholera. J. Infect. Dis. 163, 1235-1242 (1991).

80. Clemens, J. D. et al. Field trial of oral cholera vaccines in Bangladesh. Lancet 2, 124-127 (1986).

81. van Loon, F. P. et al. Field trial of inactivated oral cholera vaccines in Bangladesh: results from 5 years of follow-up. Vaccine 14, 162-166 (1996).

82. Clemens, J. et al. Field trial of oral cholera vaccines in Bangladesh: results from three-year follow-up. Lancet 335, 270-273 (1990).

83. Clemens, J. D. et al. Impact of B subunit killed whole cell and killed whole cell only oral vaccines against cholera upon treated diarrhoeal illness and mortality in an area endemic for cholera. Lancet 2, 1375-1378 (1988)

84. Sanchez, J. L. et al. Protective efficacy of oral whole-cell/recombinant-B subunit cholera vaccine in Peruvian military recruits. Lancet 344, 1273-1276 (1994)
85. Taylor, D. N. et al. Two-year study of the protective efficacy of the oral whole cell plus recombinant $B$ subunit cholera vaccine in Peru. J. Infect. Dis. 181, 1667-1673 (2000).

86. Clemens, J., Sack, D. \& Ivanoff, B. Misleading negative findings in a field trial of killed, oral cholera vaccine in Peru. J. Infect. Dis. 183, 1306-1308 (2001)

87. Lewis, D. J. et al. Immune response following oral administration of cholera toxin B subunit to HIV-1-infected UK and Kenyan subjects. AIDS 8 , 779-785 (1994).

88. Ortigão-de-Sampaio, M. B. et al. Increase in plasma viral load oral cholera immunization of HIV-infected subjects. AIDS 12, F145-F150 (1998).

89. Lucas, M. E. et al. Effectiveness of mass oral cholera vaccination in Beira, Mozambique. N. Engl. J. Med. 352, 757-767 (2005).

90. Ansaruzzaman, M. et al. Genetic diversity of El Tor strains of V. cholerae 01 with hybrid traits isolated from Bangladesh and Mozambique. Int. J. Med. Microbiol. 297, 443-449 (2007).

91. Trach, D. D. et al. Field trial of a locally produced, killed, oral cholera vaccine in Vietnam. Lancet 349, 231-235 (1997).

92. Thiem, V. D. et al. Long-term effectiveness against cholera of oral killed whole-cell vaccine produced in Vietnam. Vaccine 24, 4297-4303 (2006).

93. Anh, D. D. et al. Use of oral cholera vaccines in an outbreak in Vietnam: a case-control study. PLoS Negl. Trop. Dis. 5, e1006 (2011).

94. Trach, D. D. et al. Investigations into the safety and immunogenicity of a killed oral cholera vaccine developed in Viet Nam. Bull. World Health Organ. 80, 2-8 (2002).

95. Lopez, A. I., Clemens, J., Deen, J. \& Jodar, L. Cholera vaccines for the developing world. Hum. Vaccines 4, 165-169 (2008).

96. World Health Organization Expert Committee on Biological Standardization. Guidelines for the production and control of inactivated oral cholera vaccines. WHO Technical Report Series 924, 129-149 (2004).

97. Benenson, A. S., Saad, A. \& Mosley, W. H. Serological studies in cholera. 2 . The vibriocidal antibody response of cholera patients determined by a microtechnique. Bull. World Health Organ. 38, 277-285 (1968).

98. Mosley, W. H., Ahmad, S., Benenson, A. S. \& Ahmed, A. The relationship of vibriocidal antibody titer to susceptibility of cholera in family contacts of cholera patients. Bull. World Health Organ. 38, 777-785 (1968).

99. Anh, D. D. et al. Safety and immunogenicity if a reformulated, Vietnamese, bivalent, killed whole cell, oral cholera vaccine in adults. Vaccine $\mathbf{2 5}$, 1149-1155 (2007).

100. Mahanalabis, D. et al. A randomized, placebocontrolled trial of the bivalent killed, whole-cell cholera vaccine in adults and children in a cholera-endemic area in Kolkata, India. PLoS ONE 3, e2323 (2008).

101. Sur, D. et al. Efficacy and safety of a modified killed-whole-cell oral cholera vaccine in India: an interim analysis of a cluster-randomised, double-blind, placebo-controlled trial. Lancet 374, 1694-1702 (2009).

102. Sur, D. et al. Efficacy of a low-cost, inactivated whole-cell oral cholera vaccine: results from 3 years of follow up of a cluster-randomized, placebo-controlled trial. PLoS Negl. Trop. Dis. (in press).

103. Kaper, J. B. \& Levine, M. M. Recombinant attenuated Vibrio cholerae strains used as live oral vaccines. Res. Microbiol. 141, 901-906 (1990).
104. Cryz, S. J. Jr, Kaper, J., Tacket, C., Nataro, J. \& Levine, M. M. Vibrio cholerae CVD103-HgR live oral attenuated vaccine: construction, safety, immunogenicity, excretion and non-target effects. Dev. Biol. Stand. 84, 237-244 (1995).

105. Lagos, R. et al. Effect of small bowel bacterial overgrowth on the immunogenicity of single-dose live oral cholera vaccine CVD 103-HgR. J. Infect. Dis. 180, 1700-1712 (1999).

106. Tacket, C. O. et al. Randomized, double-blind, placebo-controlled, multicentered trial of the efficacy of a single dose of live oral cholera vaccine CVD 103-HgR in preventing cholera following challenge with Vibrio cholerae $01 \mathrm{EI}$ Tor Inaba three months after vaccination. Infect. Immun. 67, 6341-6345 (1999).

107. Suharyono, W. et al. Safety and immunogenicity of single-dose live oral cholera vaccine CVD 103-HgR in 5-9-year-old Indonesian children. Lancet 340, 689-694 (1992).

108. Su-Arehawaratana, P. et al. Safety and immunogenicity of different immunization regimens of CVD 103-HgR live oral cholera vaccine in soldiers and civilians in Thailand. J. Infect. Dis. 165, 1042-1048 (1992).

109. Tacket, C. O. et al. Onset and duration of protective immunity in challenged volunteers after vaccination with live oral cholera vaccine CVD 103-HgR. J. Infect. Dis. 166, 837-841 (1992).

110. Levine, M. M. et al. Safety, immunogenicity, and efficacy of recombinant live oral cholera vaccines, CVD 103 and CVD 103-HgR. Lancet 1, 467-470 (1988).

111. Richie, E. E. et al. Efficacy trial of single-dose live oral cholera vaccine CVD 103-HgR in North Jakarta, Indonesia, a cholera-endemic area. Vaccine 18, 2399-2410 (2000).

112. Calain, P. et al. Can oral cholera vaccination play a role in controlling a cholera outbreak? Vaccine 22, 2444-2451 (2004).

113. Perry, R. T. et al. A single dose of live oral cholera vaccine CVD $103 \mathrm{Hg}-\mathrm{R}$ is safe and immunogenic in HIV-infected and HIV-noninfected adults in Mali. Bull. World Health Organ. 76, 63-71 (1998).

114. Ali, M. et al. Herd immunity conferred by killed oral cholera vaccines in Bangladesh: a reanalysis. Lancet 366, 44-49 (2005).

115. Ali, M. et al. Vaccine protection of Bangladeshi infants and young children against cholera: implications for vaccine deployment and person-to-person transmission. Pediatr. Infect. Dis. J. 27, 33-37 (2008).

116. Longini, I. M. Jr et al. Controlling endemic cholera with oral vaccines. PLoS Med. 4, e336 (2007).

117. Jeuland, M. et al. Cost-effectiveness of newgeneration oral cholera vaccines: a multisite analysis. Value in Health 12, 899-908 (2009).

118. Vu, D. T. et al. Coverage and costs of a mass immunization of an oral cholera vaccine in Vietnam. J. Health Pop. Nutr. 21, 304-308 (2003).

119. Cavailler, P. et al. Feasibility of a mass vaccination campaign using a two dose oral cholera vaccine in an urban cholera-endemic setting in Mozambique. Vaccine 24, 4890-4895 (2006).

120. Dorlencourt, F. et al. Effectiveness of mass vaccination with $\mathrm{WC} / \mathrm{rBS}$ cholera vaccine during an epidemic in Adjumani district, Uganda. Bull. World Health Organ. 77, 949-950 (1999).

121. Chao, D. L., Halloran, M. E. \& Longini, I. M. Jr. Vaccination strategies for epidemic cholera in Haiti with implications for the developing world. Proc. Natl Acad. Sci. USA 108, 7081-7085 (2011).

122. Reyburn, R. et al. The case for reactive mass oral cholera vaccinations. PLoS Negl. Trop. Dis. 5, e952 (2011). 


\section{REVIEWS}

123. World Health Organization. Cholera vaccines: position paper. Weekly Epidemiological Record 85, 117-128 (2010).

124. Sack, D. A. et al. Evaluation of Peru-15, a new live oral vaccine for cholera, in volunteers. J. Infect. Dis. 176, 201-205 (1997).

125. Cohen, M. B. et al. Randomized, controlled human challenge study of the safety, immunogenicity, and protective efficacy of a single dose of Peru-15, a live attenuated oral cholera vaccine. Infect. Immun. 70, 1965-1970 (2002).

126. Qadri, F. et al. Randomized, controlled study of the safety and immunogenicity of Peru-15, a live attenuated oral vaccine candidate for cholera, in adult volunteers in Bangladesh. J. Infect. Dis. 192, 573-579 (2005).

127. Qadri, F. et al. Peru-15, a live-attenuated oral cholera vaccine is safe and immunogenic in Bangladeshi toddlers and infants. Vaccine $\mathbf{2 5}$ 231-238 (2007).

128. García, L. et al. The vaccine candidate Vibrio cholerae 638 is protective against cholera in healthy volunteers. Infect. Immun. 73, 3018-3024 (2005).

129. Talavera, A. et al. Process development for a Cuban cholera vaccine based on the attenuated strain Vibrio cholerae 638. Vaccine 24, 3746-3749 (2006).

130. Liu, G. et al. Resistance of the cholera vaccine candidate IEM108 against CTX $\varphi$ infection. Vaccine 24, 1749-1755 (2006).
131. Liang, W. et al. Construction and evaluation of a safe, live, oral Vibrio cholera vaccine candidate, IEM108. Infect. Immun. 71, 5498-5504 (2003).

132. Mahanalabis, D. et al. Randomized placebo controlled human volunteer trial of a live oral cholera vaccine VA1.3 for safety and immune response. Vaccine 27, 4850-4856 (2009).

133. Coster, T. S. et al. Safety, immunogenicity, and efficacy of live attenuated Vibrio cholerae 0139 vaccine prototype. Lancet 345, 949-952 (1995).

134. Tacket, C. O. et al. Initial clinical studies of CVD 112 Vibrio cholerae 0139 live oral vaccine: safety and efficacy against experimental challenge. J. Infect. Dis. 172, 883-886 (1995).

135. Ledon, T. et al. Construction and characterization of 0139 cholera vaccine candidates. Vaccine $\mathbf{2 1}$, 1282-1291 (2003).

136. Gupta, R. K., Taylor, D. N., Bryla, D. A., Robbins, J. B. \& Szu, S. C. Phase 1 evaluation of Vibrio cholerae 01, serotype Inaba, polysaccharide-cholera toxin conjugates in adult volunteers. Infect. Immun. 66, 3095-3099 (1998).

137. Boutonnier, A., Villeneuve, S., Nato, F., Dassy, B. \& Fournier, J. M. Preparation, immunogenicity, and protective efficacy, in a murine model, of a conjugate vaccine composed of the polysaccharide moiety of the lipopolysaccharide of Vibrio cholerae 0139 bound to tetanus toxoid Infect. Immun. 69, 3488-3493 (2001).

138. Kossaczka, Z. et al. Vibrio cholerae 0139 conjugate vaccines: synthesis and immunogenicity of $V$. cholerae 0139 capsular polysaccharide conjugates with recombinant diphtheria toxin mutant in mice. Infect. Immun. 68, 5037-5043 (2000)

139. Waldor, M., Hotez, P. \& Clemens, J. A national cholera vaccine stockpile-a new humanitarian and diplomatic resource. N. Engl. J. Med. 363, 2279-2282 (2010).

140. Background paper on the integration of oral cholera vaccines into global cholera control programmes. WHO strategic advisory group of experts (SAGE) on immunization [online], http://www.who.int/immunization/sage/ 1_Background_Paper_Cholera_Vaccines FINALdraft 13 oct v2.pdf (2009).

\section{Acknowledgments}

The authors thank the Bill and Melinda Gates

Foundation, and the governments of Japan, the Republic of South Korea, India, and Sweden for support. We dedicate this paper to the late Dang Duc Trach, a friend, mentor, and colleague and a major contributor to the field of cholera vaccines.

\section{Author contributions}

J. D. Clemens, S. Shin, J. Holmgren researched the data for the article. J. D. Clemens, S. Shin, D. Sur, G. B. Nair and J. Holmgren provided a substantial contribution to discussions of the content. J. D. Clemens, S. Shin, D. Sur, G. B. Nair contributed equally to the writing of the article. J. D. Clemens, S. Shin, G. B. Nair and J. Holmgren reviewed the article prior to submission. 